\title{
Gas Chromatography-Based Fingerprinting and
} \section{Chemical Pattern Recognition of Rosa multiflora Volatile} Oil

\author{
Tuniyazi Gulimeikereyi, Ablajan Nurpida, Malike Dilinuer and Hailaxi Alai \\ Department of Chemistry and Chemical Engineering, Xinjiang Normal University, Urumuqi 830054, China
}

\begin{abstract}
In this paper, gas chromatography-mass spectrometry (GS-MS) was used to build the standard fingerprint of volatile oil from Rosa multiflora Thunb. from 12 different habitats. Fourteen components in the volatile oil were identified as the indicator components of $R$. multiflora, of which one was selected as the standard. The GC analysis conditions used for fingerprinting afford a very good separating effect. The similarity of the 12 volatile oils from $R$. multiflora Thunb. was more than 0.84 , and the precision, stability and repeatability of the fingerprints were quite good. It could be concluded that the fingerprints can be used as the standard and as a quality control method for medicinal materials from $R$. multiflora Thunb..
\end{abstract}

Key words: Rosa multiflora, volatile oil, GC-MS, fingerprinting, main component.

\section{Introduction}

Recently, there has been an increased interest in drugs originating from natural sources, such as traditional Chinese medicines. More than 100,000 types of low-molecular-weight secondary metabolites are synthesized and released by plants in nature, and a significant proportion of these metabolites is volatile [1]. The components of volatile oils obtained from plants can differ with the plant's family variety, gathering season and growing region [2]. In fact, under diverse growing environments and on studying different parts of the plant [3], the same plants species can sometimes have volatile oils with different components and different contents [4, 5]. Rosa multiflora Thunb. is a member of the Rosaceae family and is also called multiflorous rosa [6]. It is widely distributed in the temperate and subtropical regions of the Northern Hemisphere, including all parts of China, where it is considered a valuable third-generation fruit. R. multiflora Thunb. [7] is used for clearing excess bodily heat and relieving toxicity, eliminating

Corresponding author: Malike Dilinuer, professor, research fields: natural products and chromatography. wing-dampness, promoting blood circulation to restore menstrual flow, securing essence and reducing urination. Each plant volatile oil has a unique fragrance, and these oils have sterilizing, stimulating and relaxing effects and can be used to generate moderate excitement and relieve fatigue. The extraction of volatile oil from plants is of great practical value to the production of spices, food industry and daily cosmetics industry, and it is also of great significance to human health.

Extraction of volatile oil is mainly carried out to concentrate the sample, eliminate interfering substances and improve the detection limits for specific compounds. The ultrasound-microwave assisted extraction (UMEA) method, which includes extraction and mechanical stirring, affords high efficiency for such extractions, because it combines the high energy of microwaves with the cavitation effect of ultrasound. Fingerprint of traditional Chinese medicine refers to using some analytical methods to mark the properly treated Chinese medicine characteristics' common peaks through the map, and grasp the characteristics of traditional Chinese 
medicine fingerprint. It effectively ensures the quality identification of traditional Chinese medicine. Chinese herbal medicine and traditional Chinese medicine quality are relatively stable, so the method is a comprehensive and quantitative appraisal method. For the evaluation of Chinese medicinal materials, the preparation quality of authenticity, excellent performance and stability are the core technology of building modern Chinese medicine quality standard system and the key to solving the drug quality control and supervision. In the present study, the volatile oil from Rosa multiflora Thunb. from 12 different habitats was extracted by the UMEA method, and gas chromatography-mass spectrometry (GC-MS) was employed to define the principal components of volatile oil for subsequent clustering analysis. The established pattern recognition method could provide a reference for the chemometry and quality testing of $R$. multiflora.

\section{Materials and Methods}

\subsection{Materials}

R. multiflora Thunb. samples gathered from Aletai, Atushi, Awati, Bole, Yili, Kashi, Xinhe, Wushi, Hetian, Bachu, Shache and the southern mountain range in Urumqi, Xinjiang (listed according to sample number) were dried in the shade and crushed.

\subsection{Sampling Conditions for UMEA}

R. multiflora Thunb. powder $(20 \mathrm{~g})$ was placed in a $500 \mathrm{~mL}$ flask with $400 \mathrm{~mL}$ of petroleum ether solution. Then, the UMEA method was usded with $276 \mathrm{~W}$ as the microwave power and $50 \mathrm{~W}$ as the ultrasound power, and the mixture was extracted for $156 \mathrm{~s}$ at room temperature [8-10]. The petroleum ether extract was then dried over anhydrous sodium sulfate, vacuum filtered, concentrated and diluted with n-hexane [11]. The diluted sample $(1 \mu \mathrm{L})$ was used for GC-MS analysis.

\subsection{Capillary GC-MS Analysis}

A56890N GC (Agilent, USA) with a flame ionization detector was used for analyzing the volatile oil. The components of the volatile oil were separated on a $30 \mathrm{~m} \times 0.25 \mathrm{~mm}$ internal diameter, diaphragm thickness $d_{f}=0.32 \mu \mathrm{m}$ HP-5 fused silica column. High purity nitrogen was used as the carrier gas at a flow rate $1.0 \mathrm{~mL} / \mathrm{min}$. The hydrogen flow rate and air flow rate were $40 \mathrm{~mL} / \mathrm{min}$ and $450 \mathrm{~mL} / \mathrm{min}$, respectively. The split ratio was 10:1, with a tail-blowing flow of $10 \mathrm{~mL} / \mathrm{min}$. The column temperature was held at $80{ }^{\circ} \mathrm{C}$, then programmed to increase at $5{ }^{\circ} \mathrm{C} / \mathrm{min}$ to $250{ }^{\circ} \mathrm{C}$, followed by gradient heating at $0.5^{\circ} \mathrm{C} / \mathrm{min}$ to $265^{\circ} \mathrm{C}$, which was held for $5 \mathrm{~min}$. Subsequently, the column was at gradient heated at $0.5^{\circ} \mathrm{C} / \mathrm{min}$ to $280{ }^{\circ} \mathrm{C}$, which was held for $5 \mathrm{~min}$. The temperature of the GC-MS inlet and ionization source was $250{ }^{\circ} \mathrm{C}$ and $300{ }^{\circ} \mathrm{C}$, respectively.

The common-mode fingerprint chromatogram was established using the average method in the software for similarity evaluation of Traditional Chinese Medicine Fingerprint Chromatograms (version 2004A). The common peak was calibrated by matching the volatile oil chromatograms of the 12 batches of $R$. multiflora Thunb. with the similarity evaluation software of the traditional Chinese medicine fingerprint chromatogram (2004A). The component that corresponded to a larger peak area and had a more stable common peak was selected as the reference substance. Data analysis was performed using SPSS 17.0, which analyzed the major components and proceeded with system clustering analysis.

\section{Results and Discussion}

\subsection{Identification of Common Components}

In accordance with the conventional definition of a fingerprint, a chromatography fingerprint is a chromatographic pattern of some of the common active components in the analyzed samples. Therefore, to build the fingerprint, it is very important to extract all the compounds present in every sample. The average chromatogram from the 12 batches was regarded as the standard characteristic fingerprint of 
volatile oil. Peaks present in all the chromatograms obtained from the 12 samples were regarded as the "common peaks" that were indicative of the similarities between the different samples. The GC chromatograms obtained from the 12 samples contained 14 distinct common peaks within $50 \mathrm{~min}$. These 14 major peaks, with a total peak area equaling $90 \%$ of the total peak area, as indicated in Fig. 1, were tentatively identified from their mass spectra. The relative retention times and relative peak areas of these common peaks are listed in Table 1.

\subsection{Validation of the Fingerprint Analysis}

\subsubsection{Precision and Repeatability Test}

To obtain a stable and repeatable chromatographic fingerprint of volatile oil for the purposes of quality control, method validation of the fingerprint analysis was performed on the basis of the relative retention times (the ratio of the retention times for the common constituent peaks to that for the reference standard peak) and relative peak areas (the ratio of the areas for the common constituent peaks to that for the reference standard peak). Among the volatile oil components of

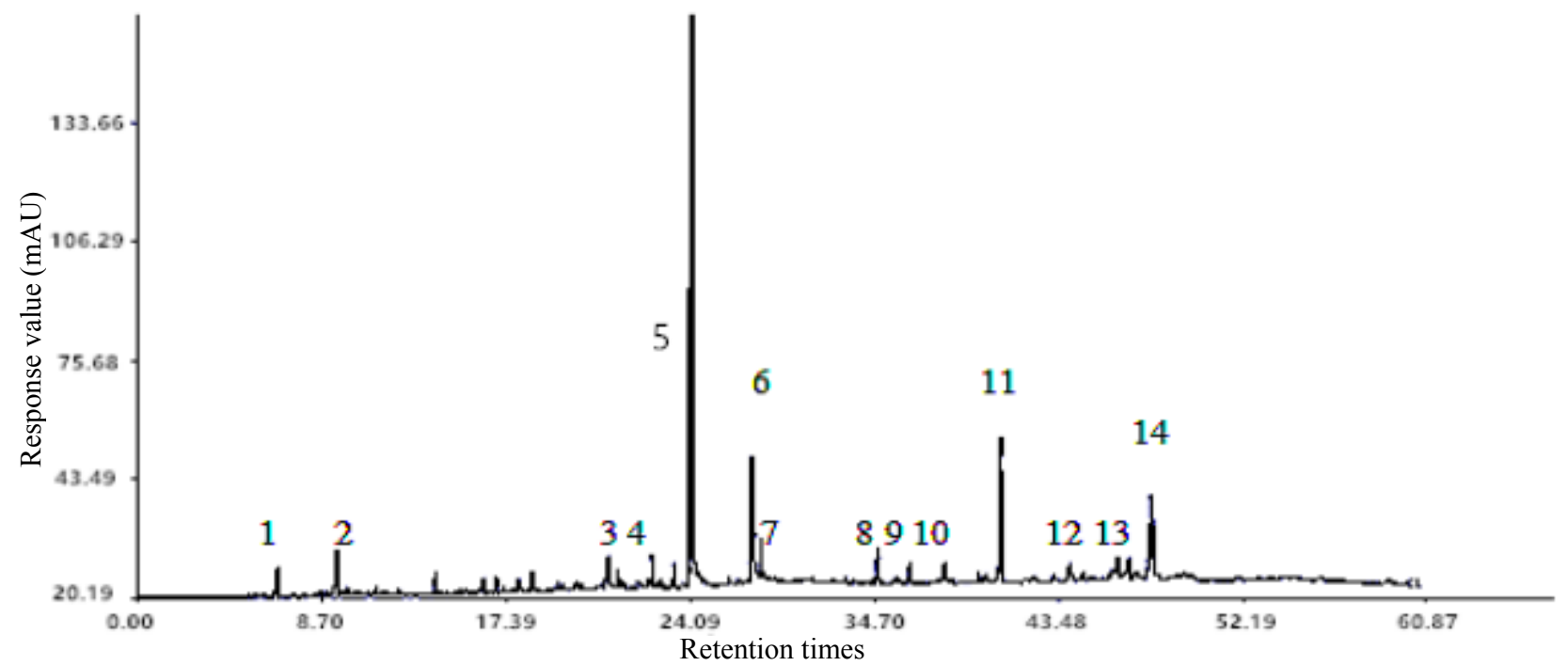

Fig. 1 Common fingerprint peaks of the volatile oil from $R$. multiflora Thunb. from 12 different habitats.

Table 1 Relative retention times and relative peak areas of the common components in 12 independently prepared samples.

\begin{tabular}{lllll}
\hline \multirow{2}{*}{ Peak No. } & \multicolumn{3}{c}{ Relative retention time } & \multicolumn{2}{c}{ Relative peak area } \\
\cline { 2 - 3 } 2 & Average & RSD (\%) & Average & RSD (\%) \\
\hline 2 & 0.248 & 0.25 & 0.0048 & 0.27 \\
3 & 0.356 & 0.15 & 0.0187 & 0.78 \\
4 & 0.867 & 1.38 & 0.0069 & 0.38 \\
5 & 0.927 & 0.11 & 0.0124 & 0.60 \\
6 & 1.000 & 0.00 & 1.0000 & 0.00 \\
7 & 1.102 & 2.35 & 0.0498 & 8.25 \\
8 & 1.130 & 2.05 & 0.1518 & 12.57 \\
9 & 1.336 & 0.22 & 0.0298 & 2.05 \\
10 & 1.393 & 0.86 & 0.0331 & 2.47 \\
11 & 1.458 & 0.73 & 0.0319 & 2.66 \\
12 & 1.559 & 0.26 & 0.1939 & 25.55 \\
13 & 1.683 & 0.16 & 0.0725 & 9.28 \\
14 & 1.787 & 0.52 & 0.0667 & 3.15 \\
\hline
\end{tabular}

RSD: relative standard deviation. 
R. multiflora Thunb., the concentration of component 5, i.e., peak 5 shown in Fig. 1, was high and stable. Consequently, this compound was chosen as the reference substance. Both the relative retention time and relative peak area of component 5 were assumed to be $100 \%$.

Injection precision was assessed by repeated injection of the same sample solution five times a day. The relative standard deviation (RSD) of the relative retention time and relative peak area was less than $0.6 \%$ and $2.57 \%$, respectively. The fingerprint repeatability was determined by analysis of the five independently prepared extracts. It is apparent from Table 2 that the RSDs of the relative retention times and relative peak areas of the 14 common components in the 12 extracts were less than $0.33 \%$ and $2.69 \%$, respectively. These results indicate that this method has high reproducibility and repeatability, and can therefore be used to build a fingerprint for volatile oil.

\subsubsection{Sample Stability}

Sample stability was assessed by analysis at $1,3,6$, 12, 24 and $48 \mathrm{~h}$ after the extraction. The results are shown in Table 3. The RSD of the relative retention times and relative peak areas of the 14 common peaks was less than $0.56 \%$ and $3.0 \%$, respectively. The similarity of these results to those from the precision and repeatability tests described above showed that the sample was stable during the period studied.

\subsubsection{Similarity Analysis}

The GC chromatograms obtained from the volatile oil extracted from $R$. multiflora Thunb. from 12 different habitats were generally similar. To differentiate between the chromatograms more clearly, it was necessary to access the similarities in the chromatographic fingerprints. The fingerprint data for the 12 samples were used to analyze the similarity among the samples by comparing each chromatogram using the similarity evaluation software of the Traditional Chinese Medicine Fingerprint Chromatogram, and a common-mode chromatogram (R) that reflected multiple chromatogram characteristics was obtained. Subsequently, the common mode (R) is compared with the 12 fingerprint chromatograms [12]. The results are shown in Table 4. The closer to 1 the correlation coefficient is, the more similar are the chromatograms. The similarity values for the $12 R$. multiflora samples were all greater than 0.90, except sample 9 and sample 11, for which the values were between 0.84 and 0.90 . It indicated that the chromatographic fingerprints obtained from the 12 samples were stable and generally consistent.

Table 2 Precision test and repeatability test.

\begin{tabular}{|c|c|c|c|c|}
\hline \multirow{2}{*}{ Peak No. } & \multicolumn{2}{|c|}{$\mathrm{RSD}(\%)$ in precision test } & \multicolumn{2}{|c|}{ RSD (\%) in repeatability test } \\
\hline & Relative retention time & Relative peak areas & Relative retention time & Relative peak areas \\
\hline 1 & 0.00 & 0.00 & 0.00 & 0.21 \\
\hline 2 & 0.00 & 0.04 & 0.00 & 1.79 \\
\hline 3 & 0.64 & 0.74 & 0.04 & 1.78 \\
\hline 4 & 0.00 & 0.00 & 0.33 & 2.55 \\
\hline 5 & 0.00 & 0.00 & 0.00 & 0.00 \\
\hline 6 & 0.05 & 1.96 & 0.00 & 0.99 \\
\hline 7 & 0.00 & 0.66 & 0.00 & 1.46 \\
\hline 8 & 0.00 & 0.44 & 0.05 & 2.52 \\
\hline 9 & 0.05 & 0.00 & 0.00 & 1.79 \\
\hline 10 & 0.00 & 0.19 & 0.00 & 1.98 \\
\hline 11 & 0.05 & 0.86 & 0.00 & 1.91 \\
\hline 12 & 0.05 & 1.42 & 0.12 & 2.69 \\
\hline 13 & 0.12 & 2.46 & 0.04 & 1.33 \\
\hline 14 & 0.04 & 2.57 & 0.05 & 2.65 \\
\hline
\end{tabular}


Table 3 Sample stability test results.

\begin{tabular}{lll}
\hline \multirow{2}{*}{ Peak No. } & \multicolumn{2}{c}{ RSD (\%) in sample stability test } \\
\cline { 2 - 3 } & Relative retention time & Relative peak area \\
\hline 1 & 0.00 & 0.06 \\
2 & 0.04 & 0.05 \\
3 & 0.00 & 0.33 \\
4 & 0.10 & 0.04 \\
5 & 0.00 & 0.00 \\
6 & 0.00 & 1.16 \\
7 & 0.00 & 0.29 \\
8 & 0.04 & 0.27 \\
9 & 0.04 & 0.05 \\
10 & 0.04 & 0.65 \\
11 & 0.56 & 2.97 \\
12 & 0.06 & 2.23 \\
13 & 0.12 & 2.99 \\
14 & 0.06 & 2.32 \\
\hline
\end{tabular}

Table 4 Similarity of the chromatograms obtained from 12 samples of $R$. multiflora Thunb. and a common-mode chromatogram.

\begin{tabular}{llllllllllllll}
\hline $\begin{array}{l}\text { Sample } \\
\text { No. }\end{array}$ & S1 & S2 & S3 & S4 & S5 & S6 & S7 & S8 & S9 & S10 & S11 & S12 & $\begin{array}{l}\text { RSD } \\
(\%)\end{array}$ \\
\hline S1 & 1.000 & 0.959 & 0.901 & 0.961 & 0.936 & 0.963 & 0.933 & 0.934 & 0.877 & 0.908 & 0.957 & 0.932 & 0.962 \\
S2 & 0.959 & 1.000 & 0.964 & 0.988 & 0.985 & 0.982 & 0.978 & 0.973 & 0.957 & 0.941 & 0.950 & 0.976 & 0.997 \\
S3 & 0.901 & 0.964 & 1.000 & 0.939 & 0.968 & 0.932 & 0.952 & 0.951 & 0.966 & 0.963 & 0.851 & 0.981 & 0.969 \\
S4 & 0.961 & 0.988 & 0.939 & 1.000 & 0.986 & 0.991 & 0.983 & 0.941 & 0.935 & 0.933 & 0.967 & 0.973 & 0.994 \\
S5 & 0.936 & 0.985 & 0.968 & 0.986 & 1.000 & 0.974 & 0.994 & 0.937 & 0.967 & 0.961 & 0.918 & 0.988 & 0.991 \\
S6 & 0.963 & 0.982 & 0.932 & 0.991 & 0.974 & 1.000 & 0.971 & 0.934 & 0.930 & 0.922 & 0.971 & 0.968 & 0.988 \\
S7 & 0.933 & 0.978 & 0.952 & 0.983 & 0.994 & 0.971 & 1.000 & 0.916 & 0.954 & 0.955 & 0.915 & 0.981 & 0.984 \\
S8 & 0.934 & 0.973 & 0.951 & 0.941 & 0.937 & 0.934 & 0.916 & 1.000 & 0.932 & 0.902 & 0.916 & 0.931 & 0.964 \\
S9 & 0.877 & 0.957 & 0.966 & 0.935 & 0.967 & 0.930 & 0.954 & 0.932 & 1.000 & 0.958 & 0.841 & 0.980 & 0.963 \\
S10 & 0.908 & 0.941 & 0.963 & 0.933 & 0.961 & 0.922 & 0.955 & 0.902 & 0.958 & 1.000 & 0.841 & 0.969 & 0.952 \\
S11 & 0.957 & 0.950 & 0.851 & 0.967 & 0.918 & 0.971 & 0.915 & 0.916 & 0.841 & 0.841 & 1.000 & 0.896 & 0.950 \\
S12 & 0.932 & 0.976 & 0.981 & 0.973 & 0.988 & 0.968 & 0.981 & 0.931 & 0.980 & 0.969 & 0.896 & 1.000 & 0.986 \\
\hline R & 0.962 & 0.997 & 0.969 & 0.994 & 0.991 & 0.998 & 0.984 & 0.964 & 0.963 & 0.952 & 0.950 & 0.986 & 1.000 \\
\hline
\end{tabular}

\subsection{Principle Component Analysis}

The statistical software SPSS 17.0 was used to analyze the correlation coefficient [13]. The results of the characteristic value and variance contribution are shown in Table 5, while Fig. 2 shows the scree plot.

The principal component was selected according to the characteristic root and variance contribution rate of the principal component. The greater the variance contribution rate, the greater the role of principal components in the analysis of sample data. Based on Fig. 2 and Table 5, the four principal components having characteristic values of more than 1 can be determined. Taking values greater than 1 as the extraction standard, the cumulative variance contribution rate reached $91.237 \%>85 \%$. This illustrates that these four components represent $91.237 \%$ of the information of the 14 components in R. multiflora Thunb. volatile oil, which is quite representative. Therefore, the first four principal components were selected to replace the original index of 14.

Table 6 shows that peaks $1,4,5,8,9,10,11,12,14$ have higher loads in principal component 1 , indicating that the 1 st principal component mainly reflects the 
Table 5 Explanation of the total variance.

\begin{tabular}{|c|c|c|c|c|c|c|}
\hline \multirow[b]{2}{*}{$\mathrm{PC}$} & \multicolumn{3}{|c|}{ Initial characteristic value and contribution } & \multicolumn{3}{|c|}{ Extracted characteristic value and contribution } \\
\hline & $\begin{array}{l}\text { Characteristic } \\
\text { value }\end{array}$ & Variance $(\%)$ & $\begin{array}{l}\text { Cumulative } \\
\text { variance }(\%)\end{array}$ & $\begin{array}{l}\text { Characteristic } \\
\text { value }\end{array}$ & Variance $(\%)$ & $\begin{array}{l}\text { Cumulative } \\
\text { variance (\%) }\end{array}$ \\
\hline 1 & 8.274 & 59.101 & 59.101 & 8.274 & 59.101 & 59.101 \\
\hline 2 & 1.851 & 13.221 & 13.221 & 1.851 & 13.221 & 13.221 \\
\hline 3 & 1.642 & 11.731 & 84.053 & 1.642 & 11.731 & 84.053 \\
\hline 4 & 1.006 & 7.184 & 91.237 & 1.006 & 7.184 & 91.237 \\
\hline 5 & 0.476 & 3.403 & 94.640 & & & \\
\hline 6 & 0.329 & 2.348 & 96.989 & & & \\
\hline 7 & 0.259 & 1.853 & 98.842 & & & \\
\hline 8 & 0.075 & 0.537 & 99.379 & & & \\
\hline 9 & 0.051 & 0.368 & 99.747 & & & \\
\hline 10 & 0.030 & 0.216 & 99.963 & & & \\
\hline 11 & 0.005 & 0.037 & 100.000 & & & \\
\hline 12 & $9.870 \times 10^{-7}$ & $7.050 \times 10^{-6}$ & 100.000 & & & \\
\hline 13 & $-3.163 \times 10^{-7}$ & $-2.260 \times 10^{-6}$ & 100.000 & & & \\
\hline 14 & $-2.747 \times 10^{-6}$ & $-1.962 \times 10^{-5}$ & 100.000 & & & \\
\hline
\end{tabular}

PC: principal components.

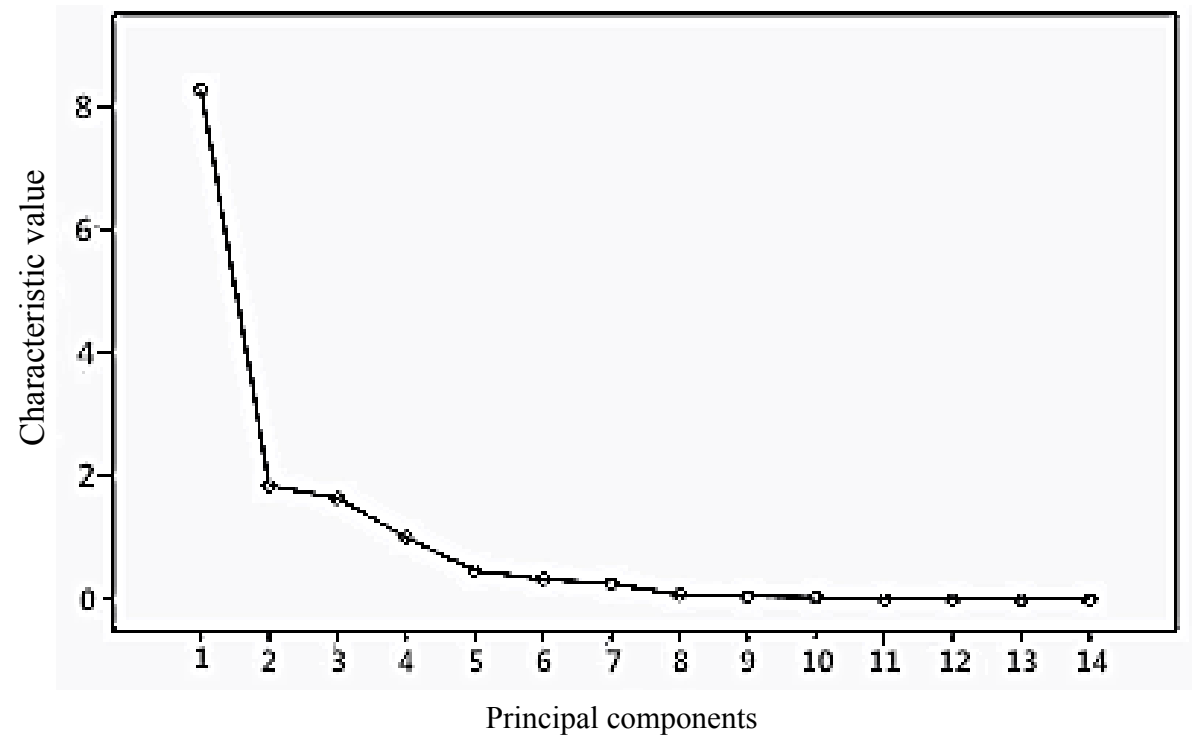

Fig. 2 Scree plot of the principal components of volatile oil .

index information of those nine components. Peak 6 has an obvious load (0.801) in principal component 2 , indicating that the 2 nd principal component mainly reflects the 6th component's index information. Peak 3 has an obvious load (0.884) in principal component 3 , indicating that the 3rd principal component mainly reflects the 3rd component's index information. Peak 2 has an obvious load (0.605) in principal component 4 , indicating that the 4 th principal component mainly reflects the 2 nd component's index information.

\subsection{Cluster Analysis}

The SPSS 17.0 software was used for cluster analysis of the 12 batches of $R$. multiflora Thunb., and the results are shown in Figs. 3 and 4. When the threshold value was $T=15$, the 12 batches of samples were divided into two category. One category contained samples 4 and 5, while the other contained the remaining 10 samples. The result of classification is consistent with the result of principal component analysis. 
Table 6 Matrix of rotated common factors.

\begin{tabular}{lllll}
\hline \multirow{2}{*}{ Peak No. } & \multicolumn{3}{c}{ Principle components } \\
\cline { 2 - 4 } & 1 & 2 & 3 & 4 \\
\hline 1 & 0.888 & -0.081 & 0.069 & -0.158 \\
2 & 0.521 & -0.130 & 0.036 & 0.605 \\
3 & 0.321 & -0.073 & 0.884 & 0.092 \\
4 & 0.830 & -0.411 & 0.305 & -0.144 \\
5 & 0.962 & -0.006 & 0.070 & -0.146 \\
6 & -0.046 & 0.801 & 0.284 & 0.429 \\
7 & 0.453 & -0.668 & 0.377 & 0.069 \\
8 & 0.933 & -0.003 & -0.266 & 0.137 \\
9 & 0.847 & -0.092 & -0.452 & 0.159 \\
10 & 0.889 & -0.152 & -0.345 & 0.194 \\
11 & 0.801 & 0.145 & -0.243 & -0.346 \\
12 & 0.814 & 0.442 & 0.256 & -0.225 \\
13 & 0.747 & 0.488 & 0.115 & -0.296 \\
14 & 0.895 & 0.280 & -0.013 & 0.191 \\
\hline
\end{tabular}

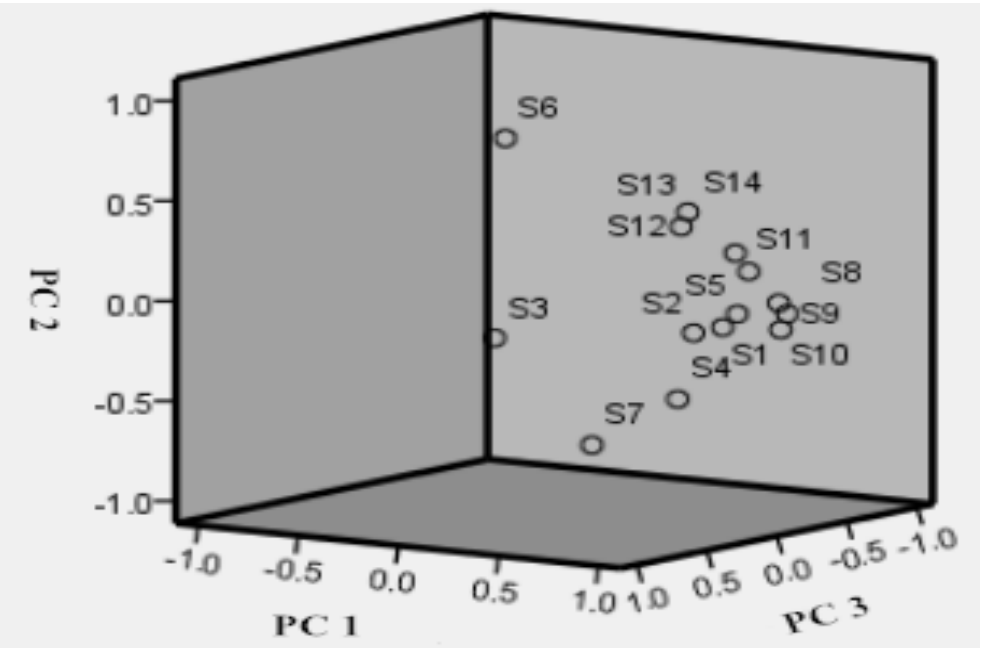

Fig. 3 3D plot of principle components.

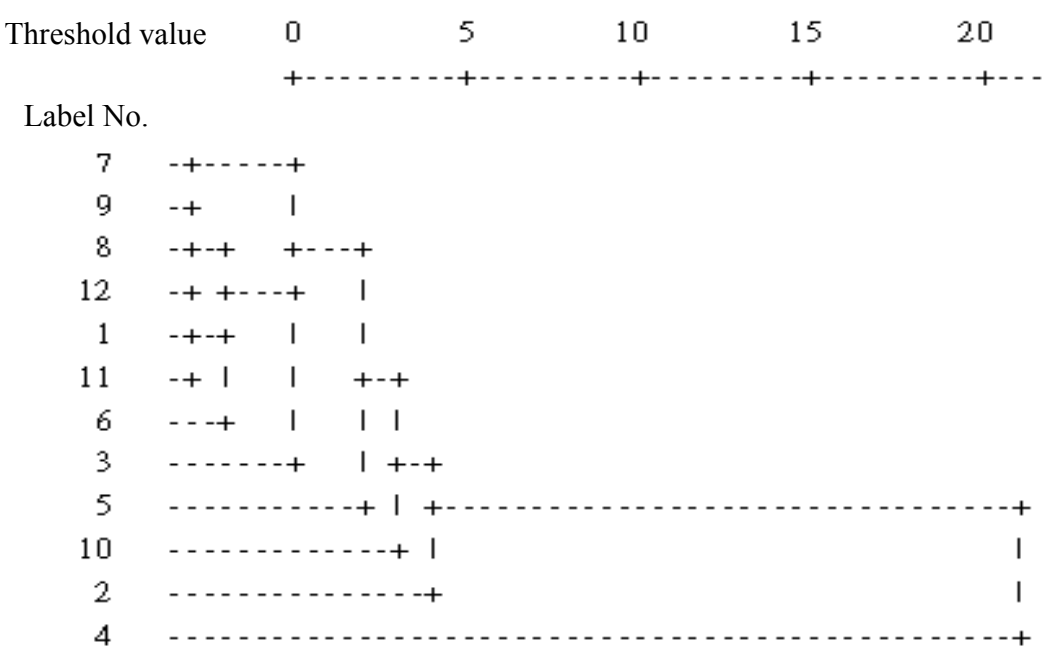

Fig. 4 Dendrogram of 12 batches of the whole herb of the sample. 


\section{Conclusions}

In this paper, volatile oil was extracted by UMEA method. Subsequently, GC was used to obtain the chemical data and the fingerprint of the volatile oil was established. Similarity evaluation, principal component analysis and cluster analysis were performed on the obtained chemical data. The similarity results showed that the similarity of the 12 batches of wild rose was more than 0.85 , which showed that samples from different habitats were somewhat similar. For cluster analysis, the 12 samples were divided into two categories, and four main components were separated based on principal component analysis. The developed fingerprinting technique has high potential for authentication of the quality of $R$. multiflora Thunb..

\section{References}

[1] Wang, W., Yuan, H. G., Chen, B., and Wu, C. J. 2006. "The Research Status of Herbal Volatile Oil." J. Lishizhen Medicine and Materia Medica Research 17 (5): 848-50. (in Chinese)

[2] Du, G. F., Cai, Z. H., Wang, G., Dai, B., and He, L. 2012. "Study on Extraction of Astragalus Polysaccharide by Ultrasonic-Microwave Synergistically Assisted Technique.” J. Nat. Prod. Res. Dev. 24: 114-7.

[3] Telci, I., Demirtas, I., and Sahin, A. 2009. "Variation in Plant Properties and Essential Oil Composition of Sweet Fennel (Foeniculum vulgare Mill.) Fruits during Stages of Maturity." Industrial Crops and Products 30 (1): 126-30.

[4] Wang, Y., Yi, L., Liang, Y., Li, H., Yuan, D., Gao, H., and Zeng, M. 2008. "Comparative Analysis of Essential Oil Components in Pericarpium Citri Reticulatae Viride and Pericarpium Citri Reticulatae by GC-MS Combined with Chemometric Resolution Method.” J. Pharm.
Biomed. Anal. 46 (1): 66-74.

[5] Zhou, X., Mo, J. G., Xie, Y. X., and Li, A. F. 2011. "Study on the Chemical Constituents of Essential Oil from Linalool Type of Cinnamomum camphorastem in Guangxi.” J. Food Sci. Tech. 36 (1): 282-5.

[6] Liu, S. X., and Cong, Z. F. 2000. Xinjiang Rose. Xinjiang, Urumqi: Xinjiang Health Science and Technology Publishing House. (in Chinese)

[7] Pang, N. N., Malike, D., and Liu, H. W. 2009. "Simultaneous Determination of Main Bioactive Components in Rosa multiflora Thunb. and Their Fragmentation Study by LC-MS." Chromatographia 70 (7-8): 1253-6.

[8] Fang, L., Chen, F. L., Yu, C. Q., Jin, Z. M., and Huang, R. P. 2013. "HPLC Fingerprint Analysis of Linderae Radix from Different Habitats." Chinese Traditional and Herbal Drugs 44 (2): 229-31. (in Chinese)

[9] Chu, G. H., Ge, Y. Q., and Ding, X. L. 2013. "HPLC Fingerprint and Its Chemical Pattern Recognition on Bramble Juice in Kashgar." Science and Technology of Food Industry 34 (6): 77-81.

[10] Zhao, H. Z., Meng, X. S., Ye, T. X., Liu, Z. H., Cheng, Y., and Luo, G. A. 2010. "HPLC Fingerprint Analysis and Chemical Pattern Recognition of Liuwei Dihuang Pills." Chinese Traditional and Herbal Drugs 41 (1): 48-51. (in Chinese)

[11] Liu, H., He, Z. J., Liang, S. W., Duan, Y. T., and Wang, Y. 2013. "Study on the HPLC Fingerprint and Chemical Pattern Recognition for Rhizome of Paris polyphylla var. yunnanensis Sampled from Different Sites." Journal of Yunnan Agricultural University 28 (1): 88-95. (in Chinese)

[12] Gu, X. F., Xin, N., Wu, H., Lin, Z. L., Liu, F. L., and Hu, Y. Z. 2011. "Pattern Recognition for Different Processed Xu Duansan Based on HPLC Fingerprints." Computers and Applied Chemistry 28 (10): 1239-43. (in Chinese)

[13] Jiang, H. F., Ou, Y. Z., Su, S. L., and Yan, H. 2011. "Study on Quality Evaluation of Medicinal Materials of Frankincense Based on Principal Components Analysis and Similarity Measure." Journal of Chinese Medicinal Materials 34 (6): 904-11. (in Chinese) 